\title{
Early Anatomical Changes and Association with Photodynamic Therapy Induced Acute Exudative Maculopathy in Patients with Macular Diseases
}

Manabu Yamamoto ( $\nabla$ shidai.srtmaia@gmail.com )

Osaka City University

Satoshi Honda

Osaka City University

Takeya Kohno

Osaka City University

Kumiko Hirayama

Osaka City University

Akika Kyo

Osaka City University

Michiko Hirabayashi

Shiraniwa Hospital

Shigeru Honda

Osaka City University

\section{Research Article}

Keywords:

Posted Date: March 7th, 2022

DOI: https://doi.org/10.21203/rs.3.rs-1379456/v1

License: (c) (i) This work is licensed under a Creative Commons Attribution 4.0 International License. Read Full License 


\section{Abstract}

The purpose of this study was to investigate the occurrence rate and predictors of photodynamic therapy (PDT) induced acute exudative maculopathy (PAEM). This retrospective study included 39 eyes of 39 patients (32 males and 7 females), who were treated with initial PDT. PAEM was defined as an increase in central retinal thickness (CRT) of 10\% or more measured by OCT on day 3 after PDT compared with baseline. Sixteen of 39 eyes (41\%) were classified in the PAEM+ group. CRT and central choroidal thickness (CCT) were significantly increased at 3 days in the PAEM+ group and significantly decreased at 1 month after PDT in the PAEM- group. In a multiple comparison, neovascular age-related macular degeneration (nAMD) had a significantly higher incidence of PAEM compared to polypoidal choroidal vasculopathy (PCV) and central serous chorioretinopathy (CSC). The incidence of PAEM was lower in PCV and CSC, and higher in nAMD. BCVA at 1 month was significantly worse in the PAEM group, which may be related to visual prognosis after PDT. Since both CRT and CCT decrease at 1 month, the detection of PAEM needs to be assessed a few days after PDT.

\section{Introduction}

Photodynamic therapy (PDT) was developed as a treatment for selective occlusion of choroidal neovascularization (CNV) and has proven to be safe and effective in preventing vision loss in neovascular age-related macular degeneration (nAMD) ${ }^{1-5}$. Since then, PDT has been applied to several macular diseases including polypoidal choroidal vasculopathy (PCV), central serous chorioretinopathy (CSC), myopic choroidal neovascularization, and choroidal hemangioma, and its efficacy has been reported ${ }^{6-9}$. As ocular complications such as choroidal circulatory disturbance, transient vision loss, extensive subretinal hemorrhage and subretinal fibrotic exudative lesions have been reported after PDT with conventional fluence and doses of verteporfin, reduced fluence or dose PDT (RF- or RD-PDT) has also been used as a method to reduce these complications ${ }^{10-16}$.

Although anti-vascular endothelial growth factor therapy (anti-VEGF) has changed the treatment of macular diseases, PDT still plays an important role ${ }^{17,18}$. In addition, in the EVEREST II trial, PDT with antiVEGF therapy for PCV showed better visual acuity improvement than PDT alone or anti-VEGF therapy alone ${ }^{6,19}$. In chronic CSC, PDT is now also considered to be the first choice over other laser treatments in meta-analyses $7,20-22$. Thus, PDT can improve vision and reduce the mental, physical, and financial burden of patients when used with sufficient consideration of its indications.

Recently, PDT-induced acute exudative maculopathy (PAEM), which develops a few days after PDT, has been reported and has attracted much attention ${ }^{23-25}$. PAEM is thought to be a condition of serous retinal detachment due to an acute fibrinous inflammatory process, which may cause vision loss in the early stage of treatment. The purpose of this study was to examine the incidence of PAEM in macular diseases and to explore the factors associated with it. 


\section{Methods}

\section{Study Participants.}

This was a retrospective, observational case series of 39 eyes of 39 patients with nAMD, PCV and CSC who underwent the initial PDT at the Department of Ophthalmology of Osaka City University Hospital between October 2016 and September 2017. This study was approved by the ethics committee of our hospital (No. 2019-062), carried out on the basis of the Declaration of Helsinki. Written informed consent was obtained from all patients prior to treatment. The mean age of patients was $72 \pm 11$ years (range, 43-90 years). Table 1 shows baseline characteristics of the patients. 17 of these 39 eyes (44\%) had undergone treatment before PDT, consisting of intravitreal ranibizumab (IVR) in 2 eyes (5\%), intravitreal aflibercept (IVA) in 9 eyes (23\%), intravitreal bevacizumab (IVB) and IVA in 1 eye (3\%), intravitreal IVR and IVA in 3 eyes (8\%), vitrectomy due to vitreous hemorrhage in 2 eyes (5\%).

Table 1

Patient characteristics at baseline.

\begin{tabular}{|ll|}
\hline Characteristics & \\
\hline Number of cases, $\mathrm{n}$ & 39 \\
\hline Male, $\mathrm{n}(\%)$ & $32(82)$ \\
\hline Age (years), Mean & $72 \pm 11$ \\
\hline Disease subtype & \\
\hline nAMD & 14 \\
\hline PCV & 14 \\
\hline CSC & 11 \\
\hline Previous treatment, $\mathrm{n}(\%)$ & $17(44)$ \\
\hline Treatment & \\
\hline Rf-PDT, $\mathrm{n}(\%)$ & $32(82)$ \\
\hline Combination, $\mathrm{n}(\%)$ & $23(59)$ \\
\hline Spot size ( $\mu \mathrm{m})$, Mean & $4623 \pm 1389$ \\
\hline BCVA (logMAR), Mean & $0.21 \pm 0.33$ \\
\hline CRT ( $\mu$ m), Mean & $334 \pm 151$ \\
\hline CCT ( $\mu$ m), Mean & $274 \pm 92$ \\
\hline
\end{tabular}

At the initial visit, all patients had their decimal best-corrected visual acuity (BCVA) measured with a Landolt $\mathrm{C}$ chart, had a fundus examination by slit-lamp biomicroscopy, fluorescein and Indocyanine green 
angiography (FA and IA) and optic coherence tomography using a confocal scanning laser ophthalmoscopy (HRA / Spectraris; Heidelberg Engineering Heidelberg, Germany). We diagnosed nAMD, PCV and CSC using OCT, FA and IA. OCT angiography was also used when the presence of CNV was suspected, which could not be confirmed by these examinations.

\section{PDT procedure.}

The PDT protocol was performed by using the full or half-fluence $\left(25 \mathrm{~J} / \mathrm{cm}^{2}\right)$ for treatment. In cases of nAMD and PCV, full-fluence was used for PDT monotherapy and half-fluence for combined anti-VEGF therapy. Half-fluence PDT was used for CSC. The verteporfin was infused over 10 minutes followed by delivery of an activating light dose of $50 \mathrm{~J} / \mathrm{cm}^{2}$ from a 689-nm laser system (Carl Zeiss, Dublin, CA) over an 83-second exposure time. The laser spot size for the PDT was the diameter of the region which was determined with FA or IA guided plus a safety zone of $500 \mu \mathrm{m}$ radius.

\section{Outcome Measures.}

Patients were evaluated by slit-lamp examination, fundus color image and OCT at baseline, 3 days and 1 month after PDT. Though PAEM is defined as a subretinal exudation with or without vision loss occurring within a few days after PDT, there are currently no clear criteria ${ }^{23,24}$. We have clearly defined PAEM to allow detection of minor changes after PDT: an increase in central retinal thickness (CRT) of $10 \%$ or more measured by OCT on day 3 after PDT compared with baseline. Patients were divided into PAEM + and PAEM- groups according to this criterion. BCVA was also measured at baseline and 1 month after PDT. The BCVA was converted to logarithm of the minimum angle of resolution (logMAR) units before analysis. Age, gender, type of disease (nAMD, PCV or CSC), PDT irradiation method (full- or half-fluence), presence of combined therapy, history of treatment before PDT, baseline BCVA, CRT, and central choroidal thickness (CCT) were selected for factors associated with the development of PAEM.

\section{Statistical Analysis.}

Changes in CRT, CCT BCVA (logMAR) from baseline were assessed using the Wilcoxon signed rank test with Bonferroni correction. In order to assess the associations between the incidence of PAEM after PDT and the various parameters, we performed univariate analyses using the chi-square test with categorial data and the Mann-Whitney U test with the parametric data. IBM SPSS Statistics 24.0 (IBM Japan, Ltd., Tokyo, Japan) was used for statistical analysis, in which $p$ values $<0.05$ were regarded as significant.

\section{Results}

Typical two cases treated with PDT are shown in Fig. 1 and Fig. 2. The subjects analyzed in this study included 39 eyes of 39 patients (32 eyes of 32 men; 7 eyes of 7 women) with nAMD, PCV and CSC. Table 1 shows baseline characteristics of the patients in this study. Mean patient age was $72 \pm 11$ years. nAMD, PCV, and CSC were included in 14, 14, and 11 eyes, respectively, of the total cases. Seventeen eyes had a history of treatment: 5 eyes were treated with ranibizumab, 14 eyes with aflibercept, 1 eye with 
bevacizumab, and 2 eyes with vitrectomy. Half-fluence PDT was performed in 32 eyes, and the combination with anti-VEGF agent was performed in 23 eyes.

Sixteen of 39 eyes (41\%) were classified in the PAEM + group. Table 2 shows changes in BCVA, CRT and CCT. In PAEM + group, mean BCVA was $0.27 \pm 0.08$ before PDT and $0.35 \pm 0.09$ after 1 month, showing a significant worsening $(p<0.05)$. In the PAEM- group, mean BCVA was $0.17 \pm 0.07$ at baseline and $0.20 \pm$ 0.07 at 1 month, showing no significant difference $(p=0.29)$. In the PAEM + group, there was a significant increase in CRT and CCT at 3 days compared to baseline, with no significant difference at 1 month (3 days, $p<0.05$ and $<0.001 ; 1$ month, $p=0.26$ and 0.48 ). In the PAEM- group, there was no significant change in CRT and CCT at 3 days, with significant decrease at 1 month ( 3 days, $p=0.17$ and 1.00; 1 month, $p<0.001$ and $<0.001)$. In the comparison between the two groups, the rate of change in CRT and CCT was significantly increased in the PAEM group at 3 days after PDT, with no significant difference at 1 month (CRT and CCT: 3 days, $p<0.05$ and $p<0.05 ; 1$ month, $p=0.17$ and $p=0.21$ ) (Fig. 3 ). In univariate analysis comparing the two groups, there were significant differences in age and clinical diagnosis as baseline factors (Age: $p<0.05$, Clinical diagnosis: $p<0.05$ ) (Table 3 ).

Table 2

Visual and anatomical changes at 3 days and 1 month after PDT compared to baseline.

\begin{tabular}{|llllll|}
\hline \multicolumn{1}{|c}{ Baseline } & 3d & $p$ Value & $1 \mathrm{M}$ & $p$ Value \\
\hline BCVA (logMAR) & & & & \\
\hline PAEM+ & $0.27 \pm 0.08$ & & & $0.35 \pm 0.09$ & $<0.05$ \\
\hline PAEM- & $0.17 \pm 0.07$ & & & $0.20 \pm 0.07$ & 0.29 \\
\hline CRT $(\mu \mathrm{m})$ & & & & \\
\hline PAEM+ & $347 \pm 39$ & $483 \pm 61$ & $<0.05$ & $285 \pm 30$ & 0.26 \\
\hline PAEM- & $325 \pm 33$ & $345 \pm 35$ & 0.17 & $196 \pm 17$ & $<0.001$ \\
\hline CCT $(\mu \mathrm{m})$ & & & & \\
\hline PAEM+ & $244 \pm 22$ & $309 \pm 22$ & $<0.001$ & $227 \pm 23$ & 0.48 \\
\hline PAEM- & $294 \pm 19$ & $303 \pm 15$ & 1.00 & $242 \pm 18$ & $<0.001$ \\
\hline
\end{tabular}


Table 3

Univariate analysis of factors associated with PAEM.

\begin{tabular}{|llll|}
\hline Characteristics & PAEM(+) & PAEM(-) & $p$ Value \\
\hline Number, $n$ & 16 & 23 & \\
\hline Male, $n(\%)$ & $13(81)$ & $19(83)$ & 0.617 \\
\hline Age (years), Mean & 76 & 69 & $<0.05$ \\
\hline $\begin{array}{l}\text { Clinical diagnosis, } n \\
\text { (nAMD : PCV : CSC) }\end{array}$ & $11: 3: 2$ & $3: 11: 9$ & $<0.05$ \\
\hline Previous treatment, $n(\%)$ & $9(56)$ & $8(35)$ & 0.158 \\
\hline Rf-PDT, n (\%) & $12(75)$ & $20(87)$ & 0.294 \\
\hline Combination, n (\%) & $10(63)$ & $13(57)$ & 0.485 \\
\hline Spot size ( $\mu$ m), Mean & $4763 \pm 1285$ & $4526 \pm 1478$ & 0.608 \\
\hline BCVA (logMAR), Mean & $0.09 \pm 0.14$ & $0.03 \pm 0.12$ & 0.172 \\
\hline CRT $(\mu m)$, Mean & $347 \pm 146$ & $325 \pm 156$ & 0.649 \\
\hline CCT $(\mu \mathrm{m})$, Mean & $244 \pm 87$ & $294 \pm 92$ & 0.100 \\
\hline
\end{tabular}

The results of comparison between the three groups according to clinical diagnosis showed significant differences in the incidence of PAEM and age (incidence of PAEM: $p<0.05$, age: $p<0.001$ ) (Table 4). In a multiple comparison of the three groups, nAMD had a significantly higher incidence of PAEM compared to PCV and CSC (nAMD versus PCV: $p<0.05$, nAMD versus CSC: $p<0.05$, PCV versus CSC: $p=1.00$ ) (Fig. 4).

Table 4

Comparison of rate of PAEM and age between disease subtype.

\begin{tabular}{|lllll|}
\hline Characteristics & nAMD & PCV & CSC & $p$ value \\
\hline Number of cases, $\mathrm{n}$ & 14 & 14 & 11 & \\
\hline Number of PAEM, $\mathrm{n}(\%)$ & $11(79)$ & $3(21)$ & $2(18)$ & $<0.05$ \\
\hline Age (years), Mean & $79 \pm 6$ & $74 \pm 6$ & $60 \pm 11$ & $<0.001$ \\
\hline
\end{tabular}

\section{Discussion}

In the present study, 16 of 37 eyes (43.2\%) were defined as PAEM, and the incidence of each disease was $79 \%$ for nAMD, $21 \%$ for PCV, and 19\% for CSC. In their study, Mammo et al. reported that in 47 CSC patients treated with PDT in 52 eyes and AMD in 5 patients treated with PDT in 6 eyes, PAEM developed in $1.4 \%$ (CSC: 1 eye, AMD: 1 eye) of the patients ${ }^{23}$. However, only patients who complained of decreased 
visual acuity a few days after the procedure were added to the examination, so it is possible that mild asymptomatic cases were missed. Manayath et al. reported on 177 patients with 155 eyes (84 sessions for CSC, 66 sessions for PCV, 5 sessions for PCV with CSC-like leakage, 15 sessions for occult CNV, and 7 sessions for posterior tumors) ${ }^{24}$. A total of 8 patients (4.52\%) had PAEM: 6 with PCV, 1 with occult CNV, and 1 with CSC. This report also suggests that PAEM without visual impairment may have been overlooked. The incidence by disease was PCV (8.45\%), occult CNV (6.67\%), and CSC (1.19\%), respectively. In this study, the definition of the onset of PAEM was strictly defined as a $10 \%$ increase in CRT early after PDT, which resulted in a more sensitive detection of PAEM, and the overall rate was higher in all diseases than previously reported. BCVA was decreased in the PAEM + group 1 month after PDT, suggesting that early vision loss may occur in those with PAEM.

Some reports have shown that PAEM is more likely to occur in CNV, followed by PCV, and rarely in chronic CSC 24,26,27. According to Holtz et al. occult CNV and classic CNV express verteporfin-targeted low-density lipoprotein receptors, which makes the RPE vulnerable to oxidative stress ${ }^{28}$. In addition, PAEM may occur in CSC because of the large choroidal vessels and the lack of components which mask RPE, but the incidence may be lower because of the absence of neovascularization ${ }^{29}$. These hypotheses are also supported by the higher incidence of PAEM in NAMD compared to PCV and CSC in this study. In addition, according to previous reports, CNV is the most likely disease to develop PAEM, followed by PCV, and it is rare in CSC. In the present study, nAMD had a higher incidence, but PCV and CSC had a lower incidence, which may be consistent with previous reports.

Although there is no clear pathogenesis for PAEM, several factors have been suggested. Schmidt et al. stated that the choroidal capillary occlusion effect of PDT causes oxidative stress in vascular endothelial cells, which stimulates histamine production and dissociation of intercellular tight junctions, resulting in increased permeability of choroidal vessels, leading to exudative changes and edema ${ }^{3}$. It has also been suggested that occlusion of the choriocapillaris after PDT causes choroidal ischemia and inflammatory changes, resulting in the production of VEGF and other substances and exudative changes in the retina and choroid ${ }^{30}$. Interestingly, in the present study, there was a significant increase in CCT as well as CRT 3 days after PDT in the PAEM + group, but not in the PAEM- group. This result may support the hypothesis previously described.

There have been reported that risk factors for the development of PAEM include age 65 years or older, PCV, BCVA 20/40 or better, PDT spot size of $350 \mu \mathrm{m}$ or larger, reduced fluence PDT, and initial PDT ${ }^{24}$. In the current study, nAMD and older age were associated with the incidence of PAEM, and there was no difference in BCVA or the method of PDT such as reduced fluence, spot size or combination therapy. Surprisingly, there was no difference in the incidence of PAEM with or without combination therapy, but this may be a point that cannot be concluded from this study due to the small sample size and differences in the selection criteria of combination therapy among disease subtypes.

In conclusion, PAEM was clearly and rigorously defined in this study, and the incidence of PAEM was lower in PCV and CSC, and higher in NAMD. BCVA at 1 month was significantly worse compared with 
baseline in the PAEM group, which may be related to visual prognosis after PDT. Since both of CRT and CCT decrease at 1 month after PDT, the detection of PAEM needs to be assessed a few days post-PDT. This study was limited by a small sample size, a non-randomized and retrospective study design and a short follow-up period. Further prospective studies with a larger number of patients will be required to confirm the association factors with PAEM found in the present study.

\section{Declarations}

\section{Data availability}

All data generated or analysed during this study are included in this published article and its supplementary information files.

\section{Author contributions statement}

M.Y., T.K. and Si.H.; methodology: M.Y. and K.T.; formal analysis: Sa.H. and M.Y.; investigation: M.Y. and T.K.; data curation: Sa.H., K.H, M.K. and M.H.; writing-original draft preparation: Sa.H. and M.Y.; and writing- review and editing: M.Y. and Si.H. All authors have read and agreed to the published version of the manuscript.

Competing interests: The authors declare no competing interests.

Funding: This study was supported in part by Grants-in-Aid for Scientific Research from the Japanese Ministry of Education, Culture, Sports, Science, and Technology (MEXT) - No.19K09997.

\section{References}

1. Schmidt-Erfurth, U. et al. Photodynamic therapy of subfoveal choroidal neovascularization: clinical and angiographic examples. Graefes Arch Clin Exp Ophthalmol 236, 365-374, doi:10.1007/s004170050092 (1998).

2. Fingar, V. H., Wieman, T. J., Wiehle, S. A. \& Cerrito, P. B. The role of microvascular damage in photodynamic therapy: the effect of treatment on vessel constriction, permeability, and leukocyte adhesion. Cancer Res 52, 4914-4921 (1992).

3. Schmidt-Erfurth, U., Laqua, H., Schlotzer-Schrehard, U., Viestenz, A. \& Naumann, G. O. Histopathological changes following photodynamic therapy in human eyes. Arch Ophthalmol 120, 835-844 (2002).

4. Barbazetto, I. et al. Photodynamic therapy of subfoveal choroidal neovascularization with verteporfin: fluorescein angiographic guidelines for evaluation and treatment-TAP and VIP report No. 2. Arch Ophthalmol 121, 1253-1268, doi:10.1001/archopht.121.9.1253 (2003).

5. Japanese Age-Related Macular Degeneration Tial Study, G. Japanese age-related macular degeneration trial: 1-year results of photodynamic therapy with verteporfin in Japanese patients with 
subfoveal choroidal neovascularization secondary to age-related macular degeneration. American Journal of Ophthalmology 136, 1049-1061, doi:10.1016/s0002-9394(03)00576-2 (2003).

6. Koh, A. et al. Efficacy and Safety of Ranibizumab With or Without Verteporfin Photodynamic Therapy for Polypoidal Choroidal Vasculopathy: A Randomized Clinical Trial. JAMA Ophthalmol 135, 12061213, doi:10.1001/jamaophthalmol.2017.4030 (2017).

7. Vasconcelos, H. et al. Long-term chorioretinal changes after photodynamic therapy for chronic central serous chorioretinopathy. Graefes Arch Clin Exp Ophthalmol 251, 1697-1705, doi:10.1007/s00417-013-2270-2 (2013).

8. Jurklies, B. \& Bornfeld, N. The role of photodynamic therapy in the treatment of symptomatic choroidal hemangioma. Graefes Arch Clin Exp Ophthalmol 243, 393-396, doi:10.1007/s00417-0041100-y (2005).

9. Varano, M., Iacono, P., Giorno, P., Chiaravalloti, A. \& Parravano, M. Photodynamic therapy in subfoveal and juxtafoveal myopic choroidal neovascularization: a 10-year retrospective analysis. Ophthalmologica 231, 204-210, doi:10.1159/000357504 (2014).

10. Sacu, S. et al. Reduced fluence versus standard photodynamic therapy in combination with intravitreal triamcinolone: short-term results of a randomised study. $\mathrm{Br} \mathrm{J}$ Ophthalmol 92, 1347-1351, doi:10.1136/bjo.2008.137885 (2008).

11. Ohkuma, Y., Hayashi, T., Sakai, T., Watanabe, A. \& Tsuneoka, H. One-year results of reduced fluence photodynamic therapy for central serous chorioretinopathy: the outer nuclear layer thickness is associated with visual prognosis. Graefes Arch Clin Exp Ophthalmol 251, 1909-1917, doi:10.1007/s00417-013-2289-4 (2013).

12. Yoshida, Y. et al. Two-year results of reduced-fluence photodynamic therapy combined with intravitreal ranibizumab for typical age-related macular degeneration and polypoidal choroidal vasculopathy. Jpn J Ophthalmol 57, 283-293, doi:10.1007/s10384-013-0234-z (2013).

13. Izumi, T. et al. Structural analyses of choroid after half-dose verteporfin photodynamic therapy for central serous chorioretinopathy. Br J Ophthalmol 101, 433-437, doi:10.1136/bjophthalmol-2016308921 (2017).

14. Nakai, S. et al. The 24-month outcomes of intravitreal aflibercept combined with photodynamic therapy for polypoidal choroidal vasculopathy. Jpn J Ophthalmol 63, 100-108, doi:10.1007/s10384018-0636-z (2019).

15. Miyamoto, N. et al. Long-term results of photodynamic therapy or ranibizumab for polypoidal choroidal vasculopathy in LAPTOP study. Br J Ophthalmol 103, 844-848, doi:10.1136/bjophthalmol2018-312419 (2019).

16. Matsumoto, H., Hoshino, J., Mukai, R., Nakamura, K. \& Akiyama, H. Short-term outcomes of intravitreal brolucizumab for treatment-naive neovascular age-related macular degeneration with type 1 choroidal neovascularization including polypoidal choroidal vasculopathy. Sci Rep 11, 6759, doi:10.1038/s41598-021-86014-7 (2021). 
17. Brown, D. M. et al. Ranibizumab versus verteporfin photodynamic therapy for neovascular agerelated macular degeneration: Two-year results of the ANCHOR study. Ophthalmology 116, 57-65 e55, doi:10.1016/j.ophtha.2008.10.018 (2009).

18. Heier, J. S. et al. Intravitreal aflibercept (VEGF trap-eye) in wet age-related macular degeneration. Ophthalmology 119, 2537-2548, doi:10.1016/j.ophtha.2012.09.006 (2012).

19. Takahashi, K. et al. Efficacy and safety of ranibizumab monotherapy versus ranibizumab in combination with verteporfin photodynamic therapy in patients with polypoidal choroidal vasculopathy: 12-month outcomes in the Japanese cohort of EVEREST II study. Clin Ophthalmol 12, 1789-1799, doi:10.2147/OPTH.S171015 (2018).

20. Lai, T. Y. et al. Safety enhanced photodynamic therapy with half dose verteporfin for chronic central serous chorioretinopathy: a short term pilot study. $\mathrm{Br} \mathrm{J}$ Ophthalmol 90, 869-874, doi:10.1136/bjo.2006.090282 (2006).

21. Tarantola, R. M., Law, J. C., Recchia, F. M., Sternberg, P., Jr. \& Agarwal, A. Photodynamic therapy as treatment of chronic idiopathic central serous chorioretinopathy. Lasers Surg Med 40, 671-675, doi:10.1002/Ism.20720 (2008).

22. Ruiz-Moreno, J. M. et al. Photodynamic therapy for chronic central serous chorioretinopathy. Acta Ophthalmol 88, 371-376, doi:10.1111/j.1755-3768.2008.01408.x (2010).

23. Mammo, Z. \& Forooghian, F. Incidence of Acute Exudative Maculopathy after Reduced-Fluence Photodynamic Therapy. Retin Cases Brief Rep 11, 217-220, doi:10.1097/ICB.0000000000000356 (2017).

24. Manayath, G. J., Ranjan, R., Vidhate, S. \& Narendran, V. PHOTODYNAMIC THERAPY-INDUCED ACUTE EXUDATIVE MACULOPATHY: Incidence, Clinical Features, and Long-Term Outcomes. Retina 40, 135144, doi:10.1097/IAE.0000000000002343 (2020).

25. Sumnicht, A. J., Chalam, K. V., Alset, A. E. \& Sierpina, D. I. The role of oral steroids in the treatment of photodynamic therapy-associated exudative maculopathy, a case report and review of the literature. Photodiagnosis Photodyn Ther 35, 102390, doi:10.1016/j.pdpdt.2021.102390 (2021).

26. Arnold, J. J. et al. Acute severe visual acuity decrease after photodynamic therapy with verteporfin: case reports from randomized clinical trials-TAP and VIP report no. 3. Am J Ophthalmol 137, 683696, doi:10.1016/j.ajo.2003.11.059 (2004).

27. Ranjan, R., Manayath, G. J. \& Vidhate, S. Exudative complications following photodynamic therapy. Can J Ophthalmol 52, 625-627, doi:10.1016/j.jcjo.2017.05.013 (2017).

28. Holz, E. R., Linares, L., Mieler, W. F. \& Weinberg, D. V. Exudative complications after photodynamic therapy. Arch Ophthalmol 121, 1649-1652, doi:10.1001/archopht.121.11.1649 (2003).

29. Somani, S. Response to "Exudative complications following photodynamic therapy". Can J Ophthalmol 52, 627-628, doi:10.1016/j.jcjo.2017.06.005 (2017).

30. Figurska, M., Wierzbowska, J. \& Robaszkiewicz, J. Severe decrease in visual acuity with choroidal hypoperfusion after photodynamic therapy. Med Sci Monit 17, CS75-79, doi:10.12659/msm.881799 (2011). 


\section{Figures}

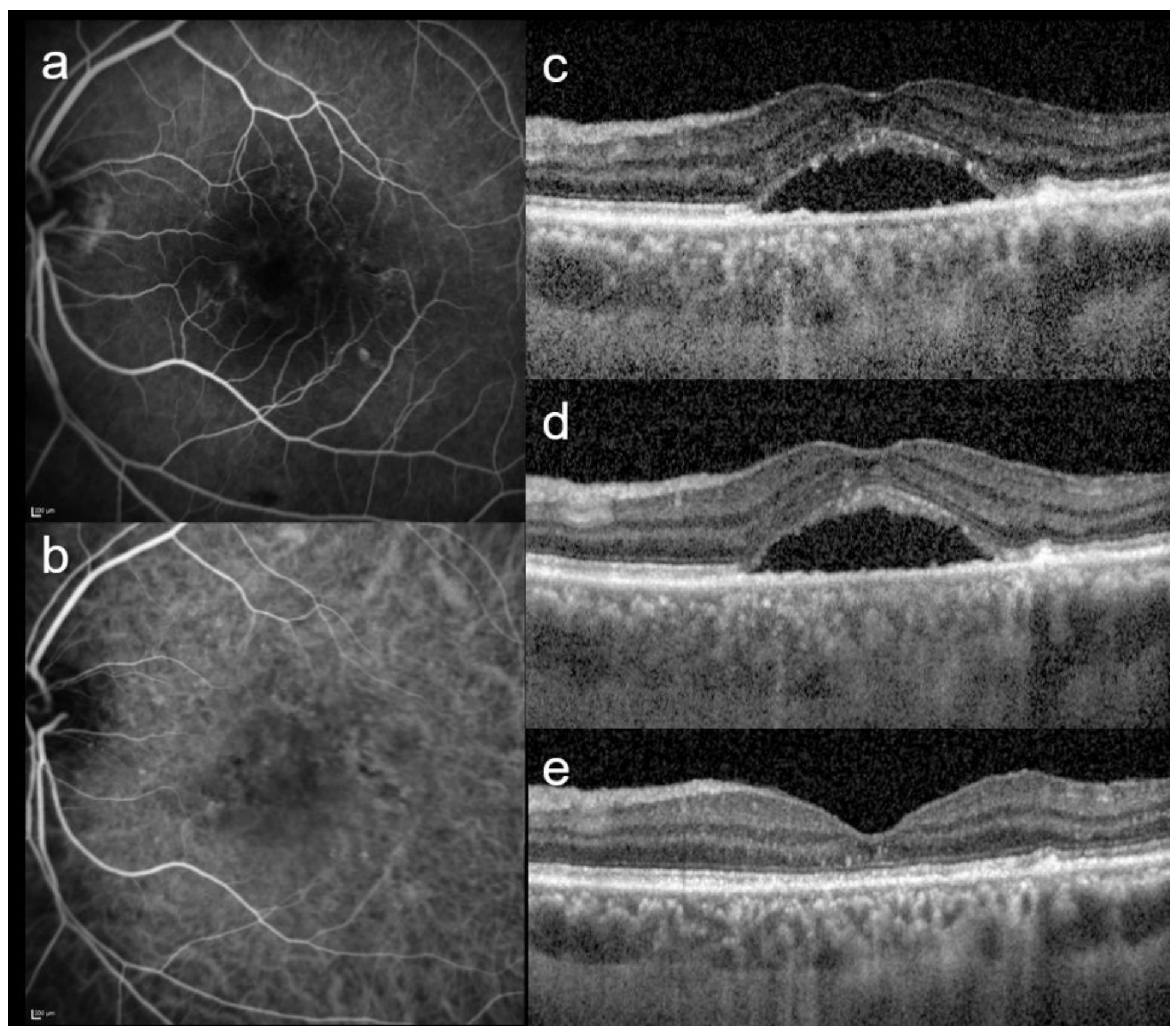

\section{Figure 1}

Fluorescein angiography (a), indocyanine green angiography (b), and horizontal line of optic coherence tomography images of patients at baseline (c), 3 days follow-up (d) and 1 month follow-up (e) after photodynamic therapy (PDT). 60-year-old-male with central serous chorioretinopathy in the left eye. Central retinal thickness was $358 \mu \mathrm{m}$ at baseline, $364 \mu \mathrm{m}(+1.7 \%)$ at 3days, $122 \mu \mathrm{m}(-65.9 \%)$ at 1 month after PDT. Central choroidal thickness was $338 \mu \mathrm{m}$ at baseline, $341 \mu \mathrm{m}(+0.9 \%)$ at 3days, $255 \mu \mathrm{m}$ $(-24.6 \%)$ at 1 month after PDT. Complete resolution of sub retinal fluid was seen at 1 month follow-up. 


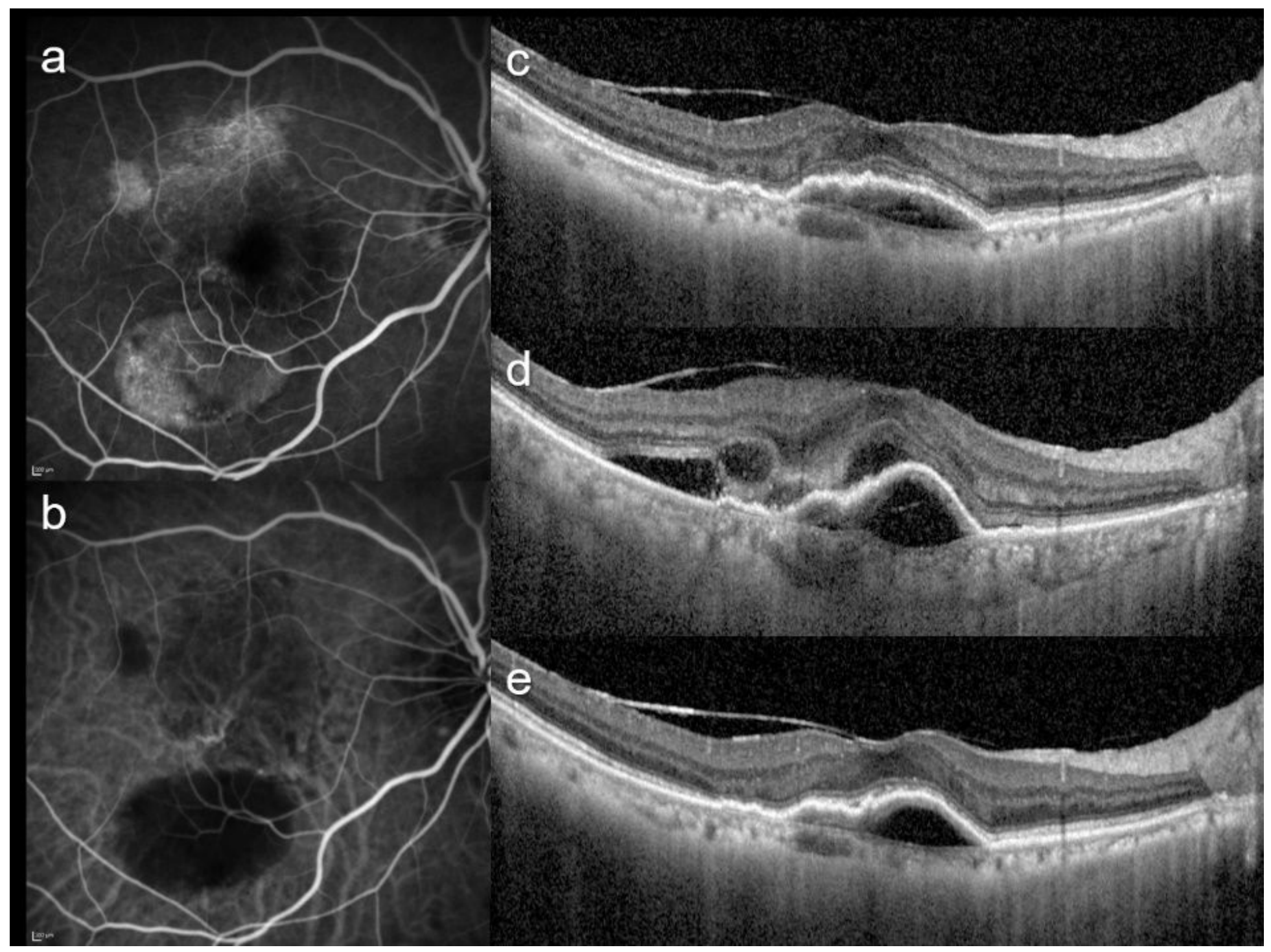

Figure 2

Fluorescein angiography (a), indocyanine green angiography (b), and horizontal line of optic coherence tomography images of patients at baseline (c), 3 days follow-up (d) and 1 month follow-up (e) after photodynamic therapy (PDT). 82-year-old-female with polypoidal choroidal vasculopathy in the light eye. Central retinal thickness was $361 \mu \mathrm{m}$ at baseline, $548 \mu \mathrm{m}(+51.8 \%)$ at 3 days, $334 \mu \mathrm{m}(-7.5 \%)$ at 1 month after PDT. Central choroidal thickness was $135 \mu \mathrm{m}$ at baseline, $270 \mu \mathrm{m}(+100.0 \%)$ at 3days, $126 \mu \mathrm{m}$ $(-6.7 \%)$ at 1 month after PDT. Complete resolution of sub retinal fluid was seen at 1 month follow-up. 

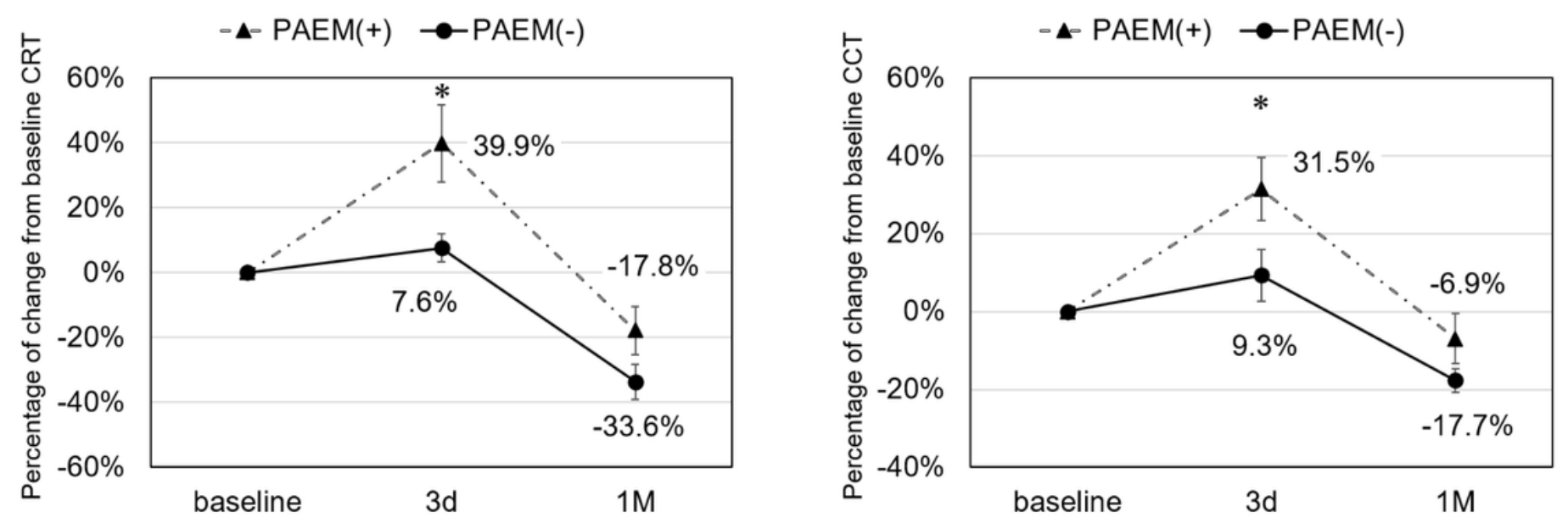

\section{Figure 3}

Change in central retinal thickness (CRT) (a) and central choroidal thickness (CCT) (b) from baseline. In the comparison between the two groups, the rate of change in CRT and CCT was significantly increased in the PAEM group at 3 days after PDT, with no significant difference at 1 month (CRT and CCT: 3 days, $p$ $<0.05$ and $p<0.05 ; 1$ month, $p=0.17$ and $p=0.21$ ).

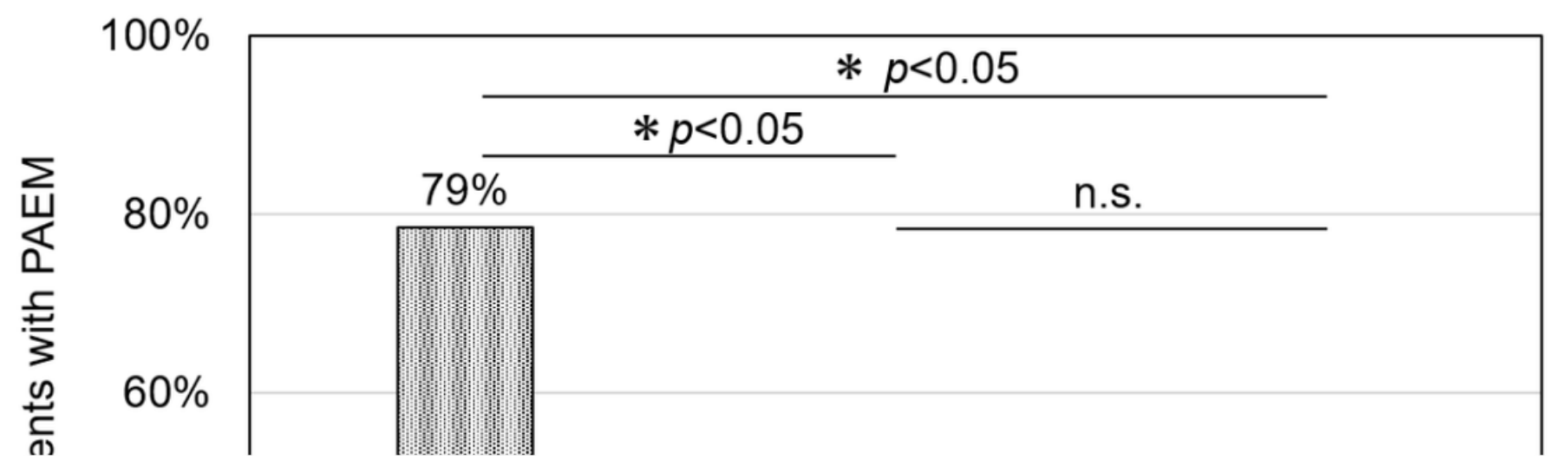


Figure 4

Incidence of PAEM by disease subtype. The incidence of PAEM was $79 \%$ in nAMD, $21 \%$ in PCV, and $19 \%$ in CSC. In a multiple comparison of the three groups, nAMD had a significantly higher incidence of PAEM compared to PCV and CSC (nAMD versus PCV: $p<0.05$, nAMD versus CSC: $p<0.05$, PCV versus CSC: $p=$ 1.00).

\section{Supplementary Files}

This is a list of supplementary files associated with this preprint. Click to download.

- supplementarymaterial.xlsx 